\title{
Quantification of within- and between-pen transmission of Foot-and-Mouth disease virus in pigs
}

\author{
Phaedra EBLÉ ${ }^{\mathrm{a} *}$, Aline DE KOEIJER ${ }^{\mathrm{b}}$, Annemarie BOUMA ${ }^{\mathrm{c}}$, \\ Arjan STEGEMAN ${ }^{\mathrm{c}}$, Aldo DEKKER ${ }^{\mathrm{a}}$ \\ ${ }^{a}$ Central Institute for Animal Disease Control (CIDC-Lelystad), PO Box 2004, \\ 8203 AA Lelystad, The Netherlands \\ ${ }^{\mathrm{b}}$ Division of Infectious Diseases, Animal Sciences Group Wageningen UR, PO Box 65, \\ 8200 AB Lelystad, The Netherlands \\ ${ }^{\mathrm{c}}$ Department of Farm Animal Health, Faculty of Veterinary Medicine, Utrecht University, Yalelaan 7, \\ 3584 CL Utrecht, The Netherlands
}

(Received 17 October 2005; accepted 1 March 2006)

\begin{abstract}
Quantified transmission parameters of Foot-and-Mouth Disease Virus (FMDV) are needed for epidemic models used for control and surveillance. In this study, we quantified the within- and between-pen transmission of FMDV in groups of pigs by estimating the daily transmission rate $\beta$, i.e. the number of secondary infections caused by one infectious pig during one day, using an SIR (susceptible-infectious-removed) model. Within-pen transmission was studied in four groups of ten pigs in which 5 infected and 5 susceptible pigs had direct contact; betweenpen transmission was studied in one group of ten pigs in which 5 infected and 5 susceptible pigs had indirect contact. Daily results of virus isolation of oropharyngeal fluid were used to quantify the transmission rate $\beta$, using Generalised Linear Modelling (GLM) and a maximum likelihood method. In addition, we estimated the expected time to infection of the first pig within a pen $T_{w}$ and in the indirect-contact pen $T_{b}$. The between-pen transmission rate $\beta_{b}$ was estimated to be 0.59 (0.083-4.18) per day, which was significantly lower than the within-pen transmission rate $\beta_{w}$ of $6.14(3.75-10.06)$. $T_{w}$ was $1.6 \mathrm{~h}$, and $T_{b}$ was $16 \mathrm{~h}$. Our results show that the transmission rate is influenced by contact structure between pigs.
\end{abstract}

foot-and-mouth disease / pigs / transmission / transmission rate / modelling

\section{INTRODUCTION}

Foot-and-Mouth disease (FMD) is a highly contagious viral disease of clovenhoofed animals, and outbreaks in unvaccinated populations can have a devastating socio-economic effect, as was seen during the outbreaks in the UK and The Netherlands in 2001. Foot-and-Mouth disease virus (FMDV) can be spread by various

\footnotetext{
*Corresponding author: phaedra.eble@wur.nl
}

ways, of which direct contact with infected animals is considered the most important, but also transmission of FMDV by slurry, transport vehicles and air-borne transmission have been described [1]. For control of the disease, it is important that the transmission of the virus be quantified. Quantitative information on transmission parameters can be used for the development of surveillance or control programmes, which e.g. can be used to determine the number 
of expected outbreaks in the high risk period of an epidemic or to evaluate which measures can reduce transmission to such a level that the virus will be eradicated. Mathematical models have for example already been used extensively during the 2001 FMD epidemic in the UK [8-10].

An often used transmission parameter is the reproduction ratio $R$, which can be used to predict if an epidemic will spread or fade out $[3,5]$. For the transmission of FMDV $R$ has been quantified previously in groups of random-mixing calves [15] and pigs [7]. A limitation of $R$, however, is that it does not include a time factor, which is important in epidemic modelling when used to analyse the course of the epidemic. Moreover, pigs in commercial pig herds are housed in pens in compartments. When using estimated within-pen parameters, the rate of transmission within a herd might be overestimated. Quantification of transmission between pens should, therefore, also be determined.

A suitable parameter to use in modelling that does have a time dimension is the transmission rate $\beta$, which is defined by the number of secondary infections caused by one infectious individual per unit of time. Although $R$ and $\beta$ are related, when the estimate of $R$ is infinite, as is often the case in a non-vaccinated population, $\beta$ cannot be derived from $R$, and therefore, another method to quantify $\beta$ is needed. In this study, we quantified the within-pen transmission of FMDV in groups of nonvaccinated pigs and between-pen transmission in a group of pigs in which the infectious and susceptible pigs only had indirect contact. The within-pen transmission rate $\beta_{w}$ was quantified using a Generalised Linear Model and the transmission rate between pens $\beta_{b}$ was quantified with a maximum likelihood method. We furthermore calculated the expected time to the first transmission in the different groups to visualise the effect of the different transmission rates.

\section{MATERIALS AND METHODS}

\subsection{Animals and experimental design}

Conventionally reared, six-week-old pigs were used in the experiments. All experiments were performed with groups of ten pigs, which were housed in animal rooms in the bio-security facilities at CIDC-Lelystad. Following an acclimatisation period of 5 days, at day 0 ( 0 dpi) five randomly selected pigs were inoculated intradermally in the bulb of the heel of the left hind-foot with $0.1 \mathrm{~mL}$ challenge virus. The remaining five pigs of each group were contact exposed to the inoculated pigs. At the time of challenge, inoculated and contact pigs were separated and the groups were reunited $24 \mathrm{~h}$ later. In the four groups in which the within-pen transmission was observed, the contact pigs were housed in the same pen as the inoculated pigs and thus had direct contact with the inoculated pigs. In the group in which the between-pen transmission was observed, the contact pigs were housed in the same animal room but were separated from the inoculated pigs by a wall of $1.50 \mathrm{~m}$ high, so that the only contact of the inoculated and contact pigs was indirect. The airflow in this experiment was directed from contact to inoculated pigs. Before the start of the experiment, approval of the Ethics Committee for Animal Experiments of CIDC-Lelystad was obtained. Buprenorfine was administered to reduce pain and pigs that suffered severely were euthanised.

\subsection{Virus}

Challenge virus contained $10^{5}$ TCID $_{50} / \mathrm{mL}$ FMDV O Taiwan (O TAW 3/97). The inoculum used was a second pig passage of material that was originally derived from the World Reference Laboratory in Pirbright, UK (O Tai 3/97, $\mathrm{RS}_{1}$ 26/3/97). 


\subsection{Sampling procedures}

Rectal temperatures and clinical signs (vesicles, lameness) of the pigs were recorded daily. After challenge, oropharyngeal fluid (OPF) was collected daily with cotton mouth-swabs, by placing a forceps with a swab in the buccal cavity for approximately $30 \mathrm{~s}$. In the laboratory, the swabs were incubated for $30 \mathrm{~min}$ in $4 \mathrm{~mL}$ EMEM containing 5\% FBS and $10 \%$ antibiotics and then centrifuged, weighed and stored at $-70{ }^{\circ} \mathrm{C}$ for virus isolation.

\subsection{Virus isolation}

OPF samples were assayed for the presence of virus by plaque titration on monolayers of secondary pig-kidney cells [4]. Ten-fold dilution series $\left(10^{0}\right.$ to $\left.10^{-2}\right)$ of the OPF samples $(200 \mu \mathrm{L}$, tested in duplicate) were allowed to adsorb for $1 \mathrm{~h}$ on monolayers of secondary pig-kidney cells in a sixwell tissue-culture plate (Greiner Bio-One $\mathrm{GmbH}$, Frickenhausen, Germany). After $1 \mathrm{~h}$, maintenance medium containing $1 \%$ methylcellulose was added. After 2 days of incubation the plates were washed in tap water with citric acid, monolayers were then rinsed with tap water and stained with amido-black $(0.1 \%$ amidoblack in $1 \mathrm{M}$ acetic acid, $0.09 \mathrm{M}$ sodium acetate, and $10 \%$ glycerol). All incubations were made at $37{ }^{\circ} \mathrm{C}$ in a humidified atmosphere containing $5 \% \mathrm{CO}_{2}$. Plaques were counted macroscopically and virus titres were expressed as ${ }^{10} \log$ plaque forming units (pfu) per $\mathrm{mL}$.

\subsection{Quantification of virus transmission}

As the basis of modelling transmission of FMDV, we used a stochastic SIR model. In an SIR model, transmission is described by the change in the number of susceptible $(S)$, infectious $(I)$, removed $(R)$ and total number $(N)$ of animals. In this model, $\mathrm{d} S / \mathrm{d} t=-\beta \cdot I(t) \cdot S(t) / N(t)$ in which $\beta$ is the transmission rate parameter. The transmission rate $\beta$ can be defined as the average number of new infections for a typical infectious animal in a susceptible population per unit of time. In the described model, the probability for a susceptible animal to escape infection during a period $\Delta t$ is given by $\mathrm{e}^{-\beta . I . \Delta t / N}$ and the probability to become infected is therefore $1-\mathrm{e}^{-\beta \cdot I \cdot \Delta \cdot t / N}$. The expected number of new infections (cases, $C$ ) is then $E(C)=S\left(1-\mathrm{e}^{-\beta \cdot I \cdot \Delta t / N}\right)$ [17].

From the experiments, using the data of the virus isolation of OPF samples, for each interval $\Delta t$ (the interval between two subsequent samplings, i.e. one day) the number of susceptible pigs at the start of the interval $(S)$, the number of infectious pigs $(I)$, the number of new cases $(C)$ and the total number of pigs $N$ are known and therefore the transmission rate $\beta$ can be estimated.

The within-pen transmission parameter $\beta_{w}$ was quantified from the above described model, using a Generalised Linear Model (GLM) [14] with a complementary loglog-LINK function and the natural logarithm of $(I \cdot \Delta t / N)$ as the offset variable. Data of the four within-pen transmission experiments were pooled and daily data of the virus isolation of the OPF samples were analysed using the GLM. Pigs were classified to be infectious from the moment that an OPF sample tested positive by virus isolation. In cases where the first contact animals tested VI-positive simultaneously with the inoculated animals, we used a half day step, assuming that the VI-positive pigs of the inoculated group were already infectious during the second half of the preceding day.

Because the GLM was inappropriate for analysis of the data of the betweenpen transmission experiment in which the inoculated-infected (subgroup A) and susceptible pigs (subgroup B) were separated by a barrier, we used a maximum 
likelihood method to quantify the betweenpen transmission. Since the probability for a susceptible animal in the susceptible subgroup (subgroup B) to become infected in time period $\Delta t$ can be given by

$$
p=1-\mathrm{e}^{-\left(\frac{\beta_{w} \cdot I_{B}(t)}{N_{B}}+\frac{\beta_{b} \cdot I_{A}(t)}{N_{A}+N_{B}}\right) \Delta t}
$$

where $\mathrm{I}_{x}(t)$ gives the number of infectious animals in subgroup $\mathrm{x}=\{\mathrm{A}, \mathrm{B}\}$ at time $t$, and $N_{x}(t)$ gives the number of animals in subgroup $x$ at time $t$, the log likelihood for $\beta_{w}$ and $\beta_{b}$ based on the daily data is

$$
\begin{aligned}
\log L\left(\beta_{w}, \beta_{b}\right)= & -\sum_{t} C \log \left(1-\mathrm{e}^{-\left(\frac{\beta_{b} I_{A}}{N_{A}+N_{B}}+\frac{\beta_{w} I_{B}}{N_{B}}\right)}\right) \\
& +(S-C)\left(\frac{\beta_{b} I_{A}}{N_{A}+N_{B}}-\frac{\beta_{w} I_{B}}{N_{B}}\right)
\end{aligned}
$$

[11] in which the previously quantified value for $\beta_{w}$ was used.

Maximising this function results in a maximum likelihood estimator for $\beta_{b}$. In order to prevent numerical problems, we applied $\beta_{b}=\exp \left(\ln \beta_{b}\right)$, thus making sure that $\beta_{b}$ became strictly positive. The $95 \%$ confidence intervals (CI) of the estimated transmission rates $\beta_{w}$ and $\beta_{b}$ were calculated assuming that asymptotically, the estimators of $\log \beta$ follow a normal distribution, and were obtained through $\ln \beta_{i} \pm$ $1.96 \operatorname{se}\left(\ln \beta_{i}\right)$. We evaluated the difference between $\ln \beta_{w}$ and $\ln \beta_{b}$ using a two-sample $t$-statistic.

To illustrate the effect of the differences between the estimated transmission rates, we also calculated the expected time to the first transmission within a pen and between pens in our experiments with the results of $\beta_{w}$ and $\beta_{b}$. Since the rate of transmission is described by $\mathrm{d} S / \mathrm{d} t=-\beta \cdot S(t) \cdot I(t) / N(t)$, the expected time to the first transmission can be given by $T=1 /\left(\beta \cdot S_{0} \cdot I_{0} / N_{0}\right)$.

\section{RESULTS}

\subsection{Clinical signs}

In the experiments studying the withinpen transmission, the first day that clinical signs (rectal temperature $>40.5{ }^{\circ} \mathrm{C}$ and/or lameness and/or vesicles) were recorded in group 1 in the inoculated pigs was $1-2 \mathrm{dpi}$ and 2-3 dpi in the contact pigs. In group 2, the inoculated pigs started to show clinical signs at 2 dpi and the contact pigs at 23 dpi. In group 3, both the inoculated and contact pigs started to show clinical signs at 2-4 dpi, and in group 4 the inoculated pigs started to show clinical signs at 23 dpi and the contact pigs at 3 dpi (Tab. I).

In the between-pen transmission group, the inoculated pigs started to show clinical signs at $2 \mathrm{dpi}$, whereas the contact pigs started to show clinical signs at 4-6 dpi (Tab. I).

\subsection{Virus isolation}

In the experiments on the within-pen transmission, virus excretion in OPF in groups 1 and 2 of the direct contact groups in the inoculated pigs started at 1dpi and at 1-2 dpi in the contact pigs. In groups 3 and 4, the inoculated pigs started to excrete virus at 1-2 dpi and the contact pigs at 2 dpi (Tab. I).

In the between-pen transmission group, the inoculated pigs started to excrete virus at 1-2 dpi, whereas the contact pigs started to excrete virus at 3-5 dpi (Tab. I).

\subsection{Quantification of virus transmission}

The within-pen transmission rate $\beta_{w}$ was estimated to be 6.14 (3.75-10.06) per day and the between-pen transmission rate $\beta_{b}$ was estimated to be 0.59 (0.083-4.18), which was significantly smaller than $\beta_{w}$ $(p<0.01)$ (Fig. 1). 
Table I. Virus isolation OPF $\left({ }^{10} \log \mathrm{pfu} / \mathrm{mL}\right)$.

A. Within-pen transmission groups.

\begin{tabular}{|c|c|c|c|c|c|c|c|c|c|c|c|c|c|c|c|}
\hline & \multicolumn{15}{|c|}{ Days post infection } \\
\hline & 0 & 1 & 2 & 3 & 4 & 5 & 6 & 7 & 8 & 9 & 10 & 11 & 12 & 13 & 14 \\
\hline \multicolumn{16}{|l|}{ Group 1} \\
\hline Inoculated & $-^{\mathrm{a}}$ & $2.0^{\mathrm{b}}$ & 4.0 & 4.2 & - & - & $\dagger^{\mathrm{c}}$ & & & & & & & & \\
\hline Inoculated & - & 2.2 & + & 4.8 & 3.3 & 2.8 & $\dagger$ & & & & & & & & \\
\hline Inoculated & - & 3.8 & 2.9 & 3.8 & - & $\dagger$ & & & & & & & & & \\
\hline Inoculated & - & 3.7 & - & 3.6 & $\dagger$ & & & & & & & & & & \\
\hline Inoculated & - & 3.8 & 1.8 & 3.2 & - & $\dagger$ & & & & & & & & & \\
\hline Contact & - & - & 2.6 & 4.7 & 4.1 & 2.8 & 2.0 & - & - & - & - & $\dagger$ & & & \\
\hline Contact & - & 3.3 & n.t. & 6.5 & 1.5 & 2.2 & - & - & $\dagger$ & & & & & & \\
\hline Contact & - & 4.3 & 4.4 & 4.0 & 3.0 & 3.0 & - & - & - & - & - & $\dagger$ & & & \\
\hline Contact & - & 2.3 & - & 4.1 & 3.4 & 3.3 & - & - & $\dagger$ & & & & & & \\
\hline Contact & - & - & 3.2 & 4.6 & 5.2 & 3.3 & 2.6 & - & - & - & - & $\dagger$ & & & \\
\hline \multicolumn{16}{|l|}{ Group 2} \\
\hline Inoculated & - & 3.4 & 3.0 & 3.4 & 3.7 & 3.3 & 2.6 & 2.8 & 2.2 & 1.8 & - & - & - & - & - \\
\hline Inoculated & - & 4.2 & 3.4 & 3.3 & 3.0 & 2.9 & 2.8 & - & 3.1 & 2.0 & - & - & - & - & - \\
\hline Inoculated & - & 2.9 & 4.8 & 3.4 & 2.9 & 1.1 & 3.0 & 2.3 & 2.1 & 2.8 & - & - & $\dagger$ & & \\
\hline Inoculated & - & 2.9 & 4.4 & 4.4 & $\dagger$ & & & & & & & & & & \\
\hline Inoculated & - & 4.3 & 3.5 & 5.0 & 3.6 & 2.2 & 2.6 & 2.9 & $\dagger$ & & & & & & \\
\hline Contact & - & - & 2.7 & 4.1 & 4.8 & 3.0 & 2.4 & 2.2 & 1.8 & 2.1 & 3.0 & - & - & - & - \\
\hline Contact & - & 2.5 & 2.1 & 4.9 & 3.2 & 2.7 & - & - & $\dagger$ & & & & & & \\
\hline Contact & - & 2.9 & 3.8 & 4.0 & 3.2 & 2.0 & 1.7 & - & - & - & - & - & - & - & - \\
\hline Contact & - & 1.8 & 3.8 & 5.1 & 3.0 & 1.8 & 1.8 & 1.7 & - & - & 2.4 & - & $\dagger$ & & \\
\hline Contact & - & 3.6 & 4.8 & 5.0 & 4.1 & 2.1 & 1.8 & - & $\dagger$ & & & & & & \\
\hline \multicolumn{16}{|l|}{ Group 3} \\
\hline Inoculated & - & - & 4.3 & 5.4 & 4.7 & $\dagger$ & & & & & & & & & \\
\hline Inoculated & - & - & 3.2 & 6.5 & 6.6 & $\dagger$ & & & & & & & & & \\
\hline Inoculated & - & - & 5.4 & 3.4 & 3.4 & $\dagger$ & & & & & & & & & \\
\hline Inoculated & - & - & 5.6 & 4.4 & 4.4 & $\dagger$ & & & & & & & & & \\
\hline Inoculated & - & 2.5 & 5.5 & 3.4 & 2.8 & $\dagger$ & & & & & & & & & \\
\hline Contact & - & - & 3.1 & 5.0 & 3.5 & $\dagger$ & & & & & & & & & \\
\hline Contact & - & - & 2.7 & 5.5 & 4.6 & 4.5 & 2.8 & 2.3 & 1.9 & - & - & 1.7 & - & - & - \\
\hline Contact & - & - & 2.8 & 5.3 & 4.0 & 4.3 & 3.3 & 1.2 & $\dagger$ & & & & & & \\
\hline Contact & - & - & 2.1 & 3.9 & 5.3 & $\dagger$ & & & & & & & & & \\
\hline Contact & - & - & 4.0 & 3.3 & 3.2 & $\dagger$ & & & & & & & & & \\
\hline \multicolumn{16}{|l|}{ Group 4} \\
\hline Inoculated & - & - & 4.1 & 4.6 & 3.3 & 2.7 & 2.1 & - & - & - & - & - & - & - & - \\
\hline Inoculated & - & 3.0 & 4.4 & 3.0 & 2.8 & $\dagger$ & & & & & & & & & \\
\hline Inoculated & - & 3.2 & 4.5 & 4.1 & $\dagger$ & & & & & & & & & & \\
\hline Inoculated & - & - & 2.6 & 5.1 & $\dagger$ & & & & & & & & & & \\
\hline Inoculated & - & - & 2.7 & 4.5 & 3.5 & $\dagger$ & & & & & & & & & \\
\hline Contact & - & - & 3.5 & 5.2 & 5.5 & 3.2 & 2.4 & - & - & - & - & - & - & - & - \\
\hline Contact & - & - & 4.6 & 4.7 & $\dagger$ & & & & & & & & & & \\
\hline Contact & - & - & 4.4 & 5.7 & 4.2 & 1.7 & 3.0 & 2.6 & $\dagger$ & & & & & & \\
\hline Contact & - & - & 3.0 & 6.2 & 3.9 & 3.4 & 1.7 & - & - & 2.5 & $\dagger$ & & & & \\
\hline Contact & - & - & 3.2 & 6.3 & 3.7 & 2.1 & 2.8 & - & $\dagger$ & & & & & & \\
\hline
\end{tabular}


Table I. Continued.

B. Between-pen transmission group.

\begin{tabular}{|c|c|c|c|c|c|c|c|c|c|c|c|c|c|c|c|}
\hline & \multicolumn{15}{|c|}{ Days post infection } \\
\hline & 0 & 1 & 2 & 3 & 4 & 5 & 6 & 7 & 8 & 9 & 10 & 11 & 12 & 13 & 14 \\
\hline \multicolumn{16}{|l|}{ Group 1} \\
\hline Inoculated & - & - & 3.3 & 3.7 & - & - & $\dagger$ & & & & & & & & \\
\hline Inoculated & - & - & 3.0 & 4.8 & 1.9 & - & - & 1.5 & 2.2 & - & - & $\dagger$ & & & \\
\hline Inoculated & - & 2.3 & + & 5.4 & - & - & - & 2.5 & $\dagger$ & & & & & & \\
\hline Inoculated & - & - & 6.3 & 3.8 & 3.5 & $\dagger$ & & & & & & & & & \\
\hline Inoculated & - & 2.5 & 4.5 & 3.4 & $\dagger$ & & & & & & & & & & \\
\hline Contact & - & - & - & - & - & 5.3 & 4.6 & 3.3 & 3.0 & 2.7 & - & $\dagger$ & & & \\
\hline Contact & - & - & - & 3.5 & 3.2 & 6.4 & 5.4 & 3.3 & 1.9 & - & - & $\dagger$ & & & \\
\hline Contact & - & - & - & - & 2.1 & 5.8 & 3.4 & 1.6 & 2.5 & $\dagger$ & & & & & \\
\hline Contact & - & - & - & - & 1.5 & 2.3 & 3.3 & 3.1 & 3.0 & - & - & $\dagger$ & & & \\
\hline Contact & - & - & - & 2.0 & 3.2 & 3.4 & 2.8 & 2.8 & - & - & - & $\dagger$ & & & \\
\hline
\end{tabular}

${ }^{a}$ No virus was isolated.

b Titre expressed as ${ }^{10} \log \mathrm{pfu} / \mathrm{mL}$.

${ }^{\mathrm{c}}$ Euthanasia piglet, if not indicated, pigs were euthanised at $14 \mathrm{dpi}$.

Titres in bold show day of start of clinical signs; + undiluted sample positive (number of plaques too many to count) but insufficient sample left to determine end-point titre.

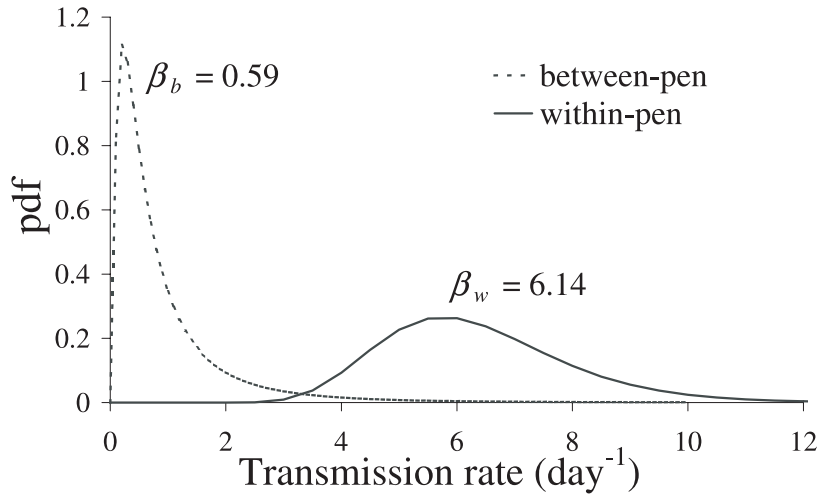

Figure 1. Probability density functions of the transmission rates $\beta$.
The expected time to infection of the first pig within a pen $T_{w}$ was estimated at $1.6 \mathrm{~h}$ whereas the expected time to infection of the first pig in the between-pen transmission pen $T_{b}$ was estimated to be $16 \mathrm{~h}$.

\section{DISCUSSION}

The purpose of this study was to quantify the within-pen and between-pen transmission rate $\beta$ of FMDV in groups of non-vaccinated pigs. The within-pen transmission rate $\beta_{w}$ was estimated to be 6.14 (3.75-10.06) per day, which was significantly larger than the $\beta_{b}$ of 0.59 (0.083-4.18). These findings indicate that the contact structure affected the transmission of FMDV significantly, and that slower spread between pens should be taken into account when the course of an FMDV infection in pig herds is analysed or modelled. It probably also means that transmission between different units and, more importantly herds, may develop 
more slowly than as estimated in withinherd transmission experiments, because the transmission of a pathogen within a herd greatly influences the transmission between herds [16].

Quantified transmission parameters can be of great use during an outbreak of FMDV to estimate the time of first introduction of the virus using the prevalence of FMDV at the time of detection, which in turn is important for forward and backward tracing. Moreover, the parameters can help in determining if and when proposed control measures will be sufficient in restricting an epidemic. Although the reduction of transmission caused by limited contact structure by itself might be insufficient to halt an epidemic, the combination with e.g. vaccination might reduce transmission sufficiently [16]. For example, we showed previously [7] that vaccination against FMDV reduced transmission of the virus significantly at $14 \mathrm{dpv}$, but not at $7 \mathrm{dpv}$. However, reduction of virus transmission within a herd might be obtained sooner than as estimated in the within-pen transmission experiments since the lower between pen transmission rate indicates that pigs in adjacent pens might have more time to develop a protective immune response.

Although we did find a significant difference in transmission rates between the within-pen and between-pen transmission groups, in all groups ultimately all contact pigs became infected and the transmission of FMDV was reduced but certainly not halted by limited contact structure. Similar results were found in studies with Classical Swine Fever [11-13]. In contrast to our results, no FMD virus transmission occurred between calves that were housed individually [2]. An explanation for this discrepancy might be that in the calf experiment only one infectious calf was used which had contact with two susceptible calves, whereas in our experiment five seeder pigs were used in a group of ten.
The results of the virus isolation of OPF of the individual pigs show, that following experimental infection of pigs, the FMD virus was highly contagious to pigs that were in direct contact, resulting in infection of all the contact pigs within 12 days. Infection of the contact pigs in the between-pen transmission group was observed after 3-5 days. In this group, probably one or two of the five contact pigs were infected by indirect transmission and the other pigs were subsequently infected by direct contact. The delay in transmission between pens is not surprising, since it is known that pigs are difficult to infect by aerosol [6].

In this study, we quantified the transmission of FMDV within- and between pens among non-vaccinated pigs. The transmission rate that we estimated can be of use in quantitative modelling which is one of the essential tools both for developing strategies in preparation for an outbreak and for predicting and evaluating the effectiveness of control policies during an outbreak. However, one should keep in mind that many parameters influence transmission, including the strain of the virus, the dose with which the pigs are infected, age, and other factors that will vary under field conditions. This variability must be taken into consideration when mathematical modelling is undertaken or veterinary advice is given for the purpose of practical disease control.

\section{ACKNOWLEDGEMENTS}

This work was supported by the European Union (FAIR5-PL97-3665) and the Ministry of Agriculture, Nature and Food Quality, The Netherlands.

\section{REFERENCES}

[1] Alexandersen S., Zhang Z., Donaldson A.I., Garland A.J.M., The pathogenesis and diagnosis of foot-and-mouth disease, J. Comp. Pathol. 129 (2003) 1-36. 
[2] Bouma A., Dekker A., De Jong M.C.M., No foot-and-mouth disease virus transmission between individually housed calves, Vet. Microbiol. 98 (2004) 29-36.

[3] De Jong M.C.M., Kimman T.G., Experimental quantification of vaccineinduced reduction in virus transmission, Vaccine 12 (1994) 761-766.

[4] De Leeuw P.W., Tiessink J.W.A., Frenkel S. Vaccination of pigs with formaldehydeinactivated aluminium hydroxide footand-mouth disease vaccines, potentiated with diethylaminoethyldextran (DEAE-D), Zentralbl. Veterinarmed. B 26 (1979) 85-97.

[5] Diekmann O., Heesterbeek J.A.P., Metz J.A.J., On the definition of and computation of the basic reproduction ratio $\mathrm{R} 0$ in models for infectious diseases in heterogeneous populations, J. Math. Biol. 28 (1990) 365-382.

[6] Donaldson A.I., Alexandersen S., Relative resistance of pigs to infection by natural aerosols of FMD virus, Vet. Rec. 148 (2001) 600-602.

[7] Eblé P.L., Bouma A., De Bruin M.G.M., van Hemert-Kluitenberg F., van Oirschot J.T., Dekker A., Vaccination of pigs two weeks before infection significantly reduces transmission of foot-and-mouth disease virus, Vaccine 22 (2004) 1372-1378.

[8] Ferguson N.M., Donnelly C.A., Anderson R.M., The foot-and-mouth epidemic in Great Britain: pattern of spread and impact of interventions, Science 292 (2001) 1155-1160.

[9] Ferguson N.M., Donnelly C.A., Anderson R.M., Transmission intensity and impact of control policies on the foot and mouth epidemic in Great Britain, Nature 413 (2001) 542-548.

[10] Keeling M.J., Woolhouse M.E.J., Shaw D.J., Matthews L., Chase-Topping M.,
Haydon D.T., Cornell S.J., Kappey J., Wilesmith J., Grenfell B.T., Dynamics of the 2001 UK foot and mouth epidemic: stochastic dispersal in a heterogeneous landscape, Science 294 (2001) 813-817.

[11] Klinkenberg D., De Bree J., Laevens H., De Jong M.C.M., Within- and between-pen transmission of classical swine fever virus: a new method to estimate the reproduction ratio from transmission experiments, Epidemiol. Infect. 128 (2001) 293-299.

[12] Laevens H., Koenen F., Deluyker H., Berkvens D., De Kruif A., An experimental infection with classical swine fever virus in weaner pigs. I. Transmission of the virus, course of the disease, and antibody response, Vet. Q. 20 (1998) 41-45.

[13] Laevens H., Koenen F., Deluyker H., De Kruif A., Experimental infection of slaughter pigs with classical swine fever virus: transmission of the virus, course of the disease and antibody response, Vet. Rec. 145 (1999) 243-248.

[14] McCullagh P., Nelder J.A., Generalized Linear Models, Chapman and Hall, London, UK, 1989

[15] Orsel K., Dekker A., Bouma A., Stegeman J.A., De Jong M.C.M., Vaccination against foot-and-mouth disease reduces virus transmission in groups of calves, Vaccine 23 (2005) 4887-4894.

[16] Van Nes A., De Jong M.C., Buijtels J.A., Verheijden J.H., Implications derived from a mathematical model for eradication of pseudorabies virus, Prev. Vet. Med. 33 (1998) 39-58.

[17] Velthuis A.G.J., De Jong M.C.M., Kamp E.M., Stockhofe N., Verheijden J.H.M., Design and analysis of an Actinobacillus pleuropneumoniae transmission experiment, Prev. Vet. Med. 60 (2003) 53-68. 